\title{
Hinweise für die Autorinnen und Autoren der ZRS
}

Die Zeitschrift für Rezensionen zur germanistischen Sprachwissenschaft hat es sich zur Aufgabe gemacht, das immer größer werdende Feld der neuen Forschungsarbeiten zu sichten und für ihre Leserinnen und Leser zugänglich zu machen. Die Zeitschrift bietet ein Forum, das in konzentrierter Form einen Überblick über aktuelle Tendenzen in der germanistischen Sprachwissenschaft gibt und so auch neue Impulse der Sprachwissenschaft nicht nur nachzeichnet, sondern über die kritische Stellungnahme auch befördert.

In der ZRS werden verschiedene Rezensionsformen gepflegt:

- die traditionelle Rezension, in der ein neuer Forschungsbeitrag besprochen und diskutiert wird,

- der review article, ein Rezensionsaufsatz, in dem eine Neuerscheinung zum Anlass genommen wird, einen weitergehenden Forschungsbeitrag in Form eines Aufsatzes zu entwickeln,

- die Sammelrezension, in der mehrere Neuerscheinungen zu einem bestimmten Themengebiet in zusammenfassender und vergleichender Weise vorgestellt werden,

- die Mehrfachrezension, bei der mehrere RezensentInnen ein Werk aus unterschiedlichen Perspektiven analysieren,

- die Replik, die die Möglichkeit der Diskussion eröffnet.

Die HerausgeberInnen haben das Recht, das Manuskript unter Wahrung der Urheberpersönlichkeitsrechte der VerfasserInnen hinsichtlich des Texts zu ändern, um die Einheitlichkeit der Zeitschrift zu gewährleisten. Die VerfasserInnen werden über solche Änderungen informiert.

Anschrift der Redaktion:

Zeitschrift für Rezensionen zur germ. Sprachwissenschaft (ZRS)

Christian-Abrechts-Universität zu Kiel | Germanistisches Seminar

Lehrstuhl für Deutsche Sprachwissenschaft

Leibnizstr. 8

D-24118 Kiel

E-Mail: zrs-kiel@germsem.uni-kiel.de 
Manuskripte sind in elektronischer Form (als E-Mail-Anhang) an die Redaktion zu senden. Für unverlangt eingesandte Manuskripte wird keine Haftung übernommen. Unaufgefordert eingesandte Rezensionsexemplare werden nicht zurückgeschickt. 